\title{
Development of a Computational Paradigm for Laser Treatment of Cancer
}

\author{
J.T. Oden ${ }^{1}$, K.R. Diller ${ }^{2}$, C. Bajaj ${ }^{3}$, J.C. Browne ${ }^{3}$, J. Hazle ${ }^{4}$, I. Babuška ${ }^{1}$, \\ J. Bass ${ }^{1}$, L. Demkowicz ${ }^{1}$, Y. Feng ${ }^{1}$, D. Fuentes ${ }^{1}$, S. Prudhomme ${ }^{1}$, \\ M.N. Rylander ${ }^{2}$, R.J. Stafford ${ }^{4}$, and Y. Zhang ${ }^{1}$ \\ ${ }^{1}$ Institute for Computational Engineering and Sciences \\ ${ }^{2}$ Department of Biomedical Engineering \\ 3 Department of Computer Science, \\ The University of Texas at Austin, Austin TX 78712, USA \\ \{oden, babuska, bass, leszek, feng, fuentes, serge, \\ jessica\}@ices.utexas.edu, \\ \{kdiller, n.forney\}@mail.utexas.edu, \\ $\{$ bajaj, browne\}@cs.utexas.edu \\ ${ }^{4}$ University of Texas M.D. Anderson Cancer Center, \\ Department of Diagnostic Radiology, Houston TX 77030, USA \\ $\{$ jhazle, jstafford $\}$ @mdanderson.org \\ http://www.ices.utexas.edu/ feng/dddas
}

\begin{abstract}
The goal of this project is to develop a dynamic data-driven planning and control system for laser treatment of cancer. The research includes (1) development of a general mathematical framework and a family of mathematical and computational models of bio-heat transfer, tissue damage, and tumor viability, (2) dynamic calibration, verification and validation processes based on laboratory and clinical data and simulated response, and (3) design of effective thermo-therapeutic protocols using model predictions. At the core of the proposed systems is the adaptive-feedback control of mathematical and computational models based on a posteriori estimates of errors in key quantities of interest, and modern Magnetic Resonance Temperature Imaging (MRTI), and diode laser devices to monitor treatment of tumors in laboratory animals. This approach enables an automated systematic model selection process based on acceptance criteria determined a priori. The methodologies to be implemented involve uncertainty quantification methods designed to provide an innovative, data-driven, patient-specific approach to effective cancer treatment.
\end{abstract}

\section{Introduction}

Today, cancer is among the leading causes of death in the United States representing $41 \%$ of the death rate in 2004 with 1.37 million new cases reported last year. According to the American Cancer Society [1, prostate cancer is responsible for $33 \%$ of the cancer deaths of male patients, and breast cancer for $32 \%$ of the cancer deaths of female patients in the United States. 
Effective cancer treatment requires complete destruction of cancerous cells while maintaining functionality of infected organs. When cancerous tumors occur in well-defined and non-vital regions, conventional surgical procedures for removal of affected tissue is the customary medical treatment; but, traditionally, little can be done when tumors are small and spread over the region. In contrast, laser surgery is minimally invasive and simple to perform, potentially decreasing complications and minimizing hospitalization. Laser therapies provide a lethal dose of heat to the desired site while minimizing damage to surrounding tissue. In particular, laser-induced hyperthermia therapies promise effective treatment of small, poorly-defined metastases or other tumors embedded within vital regions.

The success of laser treatments would be highly increased with the ability to provide a reliable prediction of treatment outcome at the time of treatment delivery using high fidelity computer predictions. Knowledge of temperature history versus time during treatment has been used to predict thermal necrosis in regions where damage is severe, but in regions where temperatures are insufficient to coagulate proteins, the results and subsequent effects have been difficult to predict. This is due, in part, to the expression of heat shock protein (HSP) in the regions of thermal stress, which provide enhanced viability of tumor tissue resulting in the recurrence of cancer. Consequently, knowledge of the thermal dose necessary to activate or de-activate HSP expression as a function of temperature and time in the affected tissue [5, 4] can be critical in planning and implementing an effective thermal treatment by laser surgery.

Grid-computing-enabled dynamic data-driven planning and control systems can provide a unique opportunity for conformal delivery of heat generated by diode or other types of lasers to the target. Image guided thermal ablation therapy surgery or as a complementary therapy for cancer management. In addition, image guidance has the potential to provide real-time treatment monitoring by providing temperature and thermal dose feedback during treatment delivery [7]. By including Magnetic Resonance Temperature Imaging (MRTI), the thermal dose delivered to surrounding normal tissue during therapy can be limited and a more conformal treatment achieved.

\section{Description of Laser Treatment Arena}

The primary objective of this paper is to describe an approach for guiding laser therapy of cancer, particularly prostate cancer, by accurate control and monitoring of the treatment process through computer simulation. This will be made possible through the development of dynamic data-driven, high-fidelity computer simulation models correlated with in vivo spatiotemporal temperature information generated during hyperthermia, and cellular and in vivo HSP expression and damage data collected to adaptively control thermo-therapy of cancerous tumors. The specific aims supporting this objective are:

1. To develop an adaptive control system that operates over a computational grid connecting a Treatment/Measurement Arena (TMA) in Houston at 
the UT's M.D. Anderson Cancer Center (MDACC) and a Computational/ Simulation Arena (CSA) in Austin at The University of Texas at Austin (UT Austin).

2. To develop new algorithms, laboratory and modeling protocols to enable the development of the control systems, including adaptive modeling and meshing procedures, calibration procedures, verification and validation procedures, inverse modeling and sensitivity analysis algorithms, and laboratory procedures for measuring tissue damage and HSP expressions to characterize the kinetic relationship in terms of temperature and time.

3. To demonstrate the effectiveness of the entire process by applying it to the treatment of actual prostate tumors in canines, using modern MRTI-guided laser surgery, distributed visualization and imaging techniques, and data storage and processing devices.

This paper will focus on the mathematical characterization of HSP expression, mesh generation, and development of the computational infrastructure. Development of the control system to operate over a computational grid will be discussed in future work.

\section{HSP Characterization and Damage Model}

Heat Shock Proteins are a family of gene products expressed in higher concentrations in the presence of environmental stresses. The name vastly understates the HSP family's astounding versatility. These proteins have been identified as critical components of cell survival under adverse environmental conditions. Various lev-

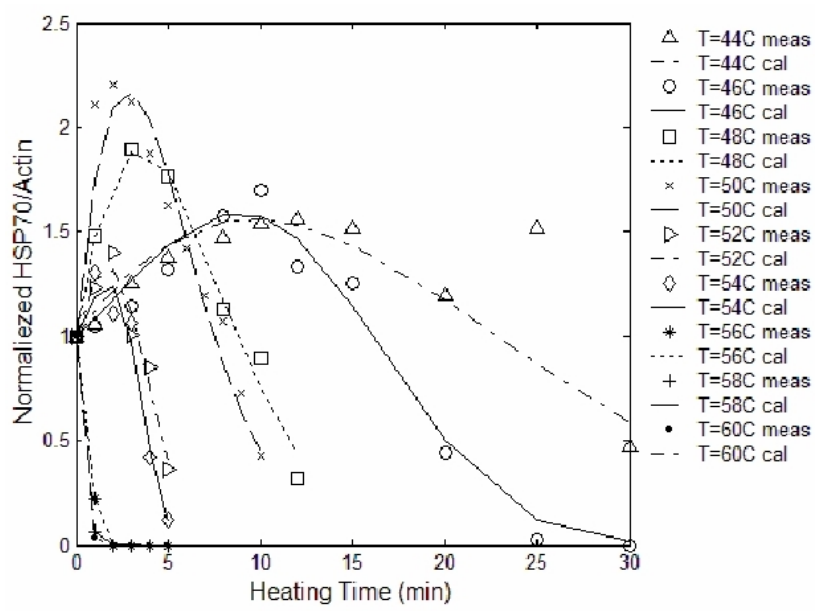

Fig. 1. HSP Expression Model els of HSP species indicate the health or likelihood of cell proliferation or drug resistance. Also, measures of cell damage as a function of temperature and time for a specific patient is a critical indication of the effectiveness of thermotherapies. Knowledge of the temperatures necessary to elicit interaction in tumor resistance and cell damage is essential to effectively produce a desired tissue response and surgical outcome. 
The work of Rylander 4] developed a model for HSP expression and cell damage based on an Arrhenius model for a mouse. Figure1 shows the comparison of the experimentally determined HSP expression with the predicted values of (1). According to Rylander [4], HSP expression $H(T, t)$ can be predicted by:

$$
H(T, t)=H_{0} e^{\alpha t-\beta t^{\gamma}}
$$

where $\alpha, \beta$, and $\gamma$ are time independent parameters that may depend on temperature, with $\gamma>1$.

Cellular damage is measured in terms of the damage fraction $F_{D}$ predicted by means of an Arrhenius integral formulation [5].

$$
F_{D}(t)=1-e^{-\Omega(t)} \quad \Omega(t)=\ln \left(C_{0} / C_{t}\right)=A \int_{0}^{t} e^{E_{a} / R T(\tau)} d \tau
$$

where $C_{0}$ is the initial concentration of healthy cells, $C_{t}$ the concentration of healthy cells after heating at time $t, A$ the pre-exponential scaling factor, $E_{a}$ the activation energy of the injury process, $R$ the universal gas constant, and $T$ the absolute temperature.

\section{The Bioheat Transfer Model}

Driving the prediction of the HSP and cellular damage is the temperature field produced by the library of bioheat models. The work of Liu 2 has shown Pennes model [3] to give good results for prediction of temperature field in the prostate. As a result only Pennes model will be discussed. Determination of other feasible vasculature and/or continuum bioheat transfer models is in progress.

A space-time variational construction of Pennes equation is used for developing the optimal control framework and in the computational implementation. Following the standard procedure and assuming Cauchy boundary conditions, the variational form of Pennes model is:

$$
\begin{array}{r}
\text { Find } T(\mathbf{x}, t) \in L^{2}\left([0, \tau], H^{1}(\Omega)\right) \text { with } T^{\prime}(\mathbf{x}, t) \in L^{2}\left([0, \tau], L^{2}(\Omega)\right): \\
B(T ; v)=F(v) \quad \forall v \in L^{2}\left([0, \tau], H^{1}(\Omega)\right)
\end{array}
$$

where

$$
\begin{gathered}
B(T ; v)=\int_{0}^{\tau} \int_{\Omega}\left[\rho c_{p} \frac{\partial T}{\partial t} v+k \nabla T \cdot \nabla v-\omega c_{\text {blood }}\left(T_{a}-T\right) v\right] d x d t \\
-\int_{0}^{\tau} \int_{\partial \Omega} h T v d A d t+\int_{\Omega} T(\mathbf{x}, 0) v(\mathbf{x}, 0) d x \\
F(v)=\int_{0}^{\tau} \int_{\Omega} Q_{\text {laser }}(\mathbf{x}) v d x d t-\int_{0}^{\tau} \int_{\partial \Omega} h T_{\infty} v d A d t+\int_{\Omega} T^{0} v(\mathbf{x}, 0) d x
\end{gathered}
$$

Here $k$ and $\omega$ are bounded functions of $\mathrm{T}$, linear in the temperature regimes of interest, $c_{p}$ and $c_{\text {blood }}$ are the specific heats, $T_{a}$ the arterial temperature, and $h$ is the coefficient of cooling. The laser source term $Q_{\text {laser }}$ is a linear function of laser power and exhibits exponential decay with distance from the source. See [5] for additional details. 


\section{Mesh Generation}

The first step in simulation is to construct a finite element mesh used for the governing equations within the library of bioheat transfer models. The mesh generation involves two parts: (1) construction, from MRI data, of a finite element mesh to represent the geometry (2) overlaying the MRTI temperature field onto the finite element mesh.

Prostate tumor cells were inoculated in the hind legs of a mouse and grown to a tumor burden of less than $1.0 \mathrm{cc}$, Figure 2, A sample of the MRI data used to construct a mesh of the mouse and tumor is shown in Figure 3. The hexahedral mesh of the tumor (blue) and tissue (yellow), shown in Figure 2, was created by a semi-automatic segmentation method adapted to find the interface boundaries of tumor and tissue. $\mathrm{Cu}-$ bic spline and lofting methods are applied to obtain smooth boundaries from the segmented MRI data [5, 8,

Spatio-temporal temperature distribution is measured during the laser treatment with update times less than 5 seconds per image
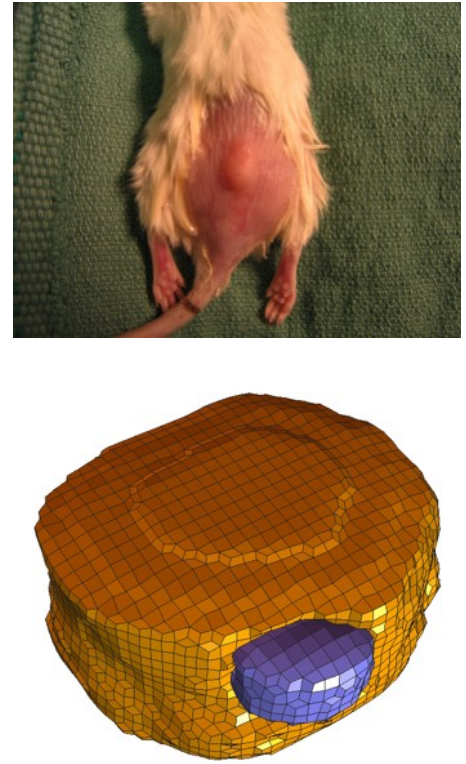

Fig. 2. Mouse and Mesh (from [5] and thickness between planes of $2.0 \mathrm{~mm}$. The temperature field, measured in a canine prostate, is accurately overlaid onto the geometry data and nodal temperature values are assigned to the mesh by taking the interpolant of the MRTI temperature data.
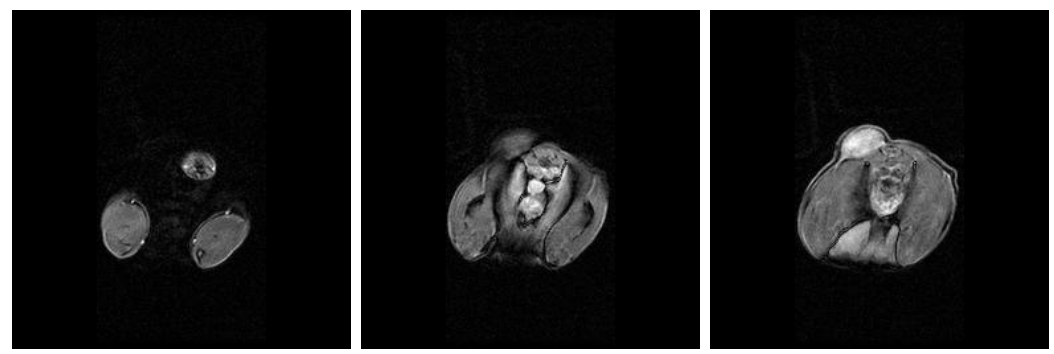

Fig. 3. MRI Data of Mouse

\section{Control System}

The dynamic flow of every problem posed within the laser treatment control system may be cast under the following mathematical framework. 


$$
\begin{aligned}
& \text { Find } \xi \in \mathcal{W}: Q(\xi)=\inf _{\eta \in \mathcal{M}} Q(\eta) \\
& \qquad \mathcal{M}=\{\eta \in \mathcal{W}: B(\eta ; v)=F(v) \quad v \in \mathcal{V}\}
\end{aligned}
$$

where $Q: \mathcal{W} \rightarrow \mathbb{R}$ is a functional characterizing a particular quantity of interest and a saddle point, $(\xi, p) \in \mathcal{W} \times \mathcal{V}$ of the Lagrangian

$$
L(\xi ; p)=Q(\xi)+F(p)-B(\xi ; p)
$$

solves the optimal control problem. Thus the problems of calibration, optimization, and computing local computing local error estimates dynamically for each model within the library of bioheat transfer models are to solve:

$$
\begin{aligned}
\text { Find }(\xi, p) \in \mathcal{W} \times \mathcal{V} & \\
B(\xi ; v) & =F(v) \quad \forall v \in \mathcal{V} \\
B^{\prime}(\xi ; \eta, p) & =Q^{\prime}(\xi, \eta) \quad \forall \eta \in \mathcal{W}
\end{aligned}
$$

Algorithms for discretizing and solving the dynamic calibration and optimization problems and dynamically computing local error estimates may be studied through a single example; we consider here the calibration problem. The problem of model calibration is to find the set of model parameters that minimize the $L_{2}$ norm of the difference between the predicted temperature field and the experimentally determined temperature field at each time instance of the experimental data. The calibration problem may be stated as follows:

Given experimentally determined temperature fields at times $t_{1}, t_{2}, \ldots, t_{n}$

$$
T_{e x p}^{t_{1}}(\mathbf{x}), T_{e x p}^{t_{2}}(\mathbf{x}), \ldots \ldots, T_{e x p}^{t_{n}}(\mathbf{x})
$$

Find the temperature field, $T^{*}$, and best combination of model coefficients, $\beta^{*}=\left(k_{0}^{*}, k_{1}^{*}, \omega_{0}^{*}, \omega_{1}^{*}\right)$, that minimizes

$$
Q(T, \beta)=\sum_{k} \int_{\Omega}\left(T\left(\mathbf{x}, t_{k}\right)-T_{e x p}^{t_{k}}(\mathbf{x})\right)^{2} d x
$$

i.e. $\left(T^{*}, \beta^{*}\right)$ satisfies

$$
\begin{aligned}
Q\left(T^{*}, \beta^{*}\right) & =\inf _{(T, \beta) \in \mathcal{M}} Q(T, \beta) \\
& \mathcal{M}=\{(T, \beta) \in \mathcal{W}: B(T, \beta ; v)=F(v) \quad \forall v \in \mathcal{V}\}
\end{aligned}
$$

The semilinear and linear forms are defined from (2) with $k=k\left(T, k_{0}, k_{1}\right)$ and $\omega=\omega\left(T, \omega_{0}, \omega_{1}\right)$. 
For appropriately chosen test functions, a solution $(T, \beta, p)$ to the calibration problem satisfies the forward in time state equation with initial conditions,

$$
\begin{aligned}
\int_{\Omega} & {\left[\rho c_{p} \frac{\partial T}{\partial t} v+k\left(T, k_{0}, k_{1}\right) \nabla T \cdot \nabla v-\omega\left(T, \omega_{0}, \omega_{1}\right) c_{b l o o d}\left(T_{a}-T\right) v\right] d x d t } \\
& -\int_{\partial \Omega} h T v d A d t=\int_{\Omega} Q_{l a s e r}(\mathbf{x}) v d x d t-\int_{\partial \Omega} h T_{\infty} v d A d t \quad \forall v \\
\quad+\text { initial conditions } &
\end{aligned}
$$

a backward in time adjoint/dual equation with terminal conditions ,

$$
\begin{gathered}
\int_{\Omega}-\rho c_{p} \frac{\partial p}{\partial t} \hat{T}+k\left(T, k_{0}, k_{1}\right) \nabla \hat{T} \cdot \nabla p+\hat{T} \frac{\partial k}{\partial T} \nabla T \cdot \nabla p d x d t \\
+\int_{\Omega} \omega\left(T, \omega_{0}, \omega_{1}\right) \hat{T} p+\hat{T} \frac{\partial \omega}{\partial T}\left(T_{a}-T\right) p d x d t \\
\quad=2 \sum_{k} \int_{\Omega}\left(T\left(\mathbf{x}, t_{k}\right)-T_{e x p}^{t_{k}}\right) \hat{T}\left(\mathbf{x}, t_{k}\right) d x \quad \forall \hat{T} \\
\quad+\text { terminal conditions }
\end{gathered}
$$

and a control equation, which may be shown to be the gradient of the quantity of interest/objective function.

$$
\nabla_{\beta} Q=\left[\begin{array}{c}
\int_{0}^{\tau} \int_{\Omega} \frac{\partial k}{\partial k_{0}} \nabla T \cdot \nabla p d x d t \\
\int_{0}^{\tau} \int_{\Omega} \frac{\partial k}{\partial k_{1}} \nabla T \cdot \nabla p d x d t \\
\int_{0}^{\tau} \int_{\Omega} \frac{\partial \omega}{\partial \omega_{0}}\left(T_{a}-T\right) p d x d t \\
\int_{0}^{\tau} \int_{\Omega} \frac{\partial \omega}{\partial \omega_{1}}\left(T_{a}-T\right) p d x d t
\end{array}\right]=\mathbf{0}
$$

For bioheat models involving larger parameter sets $(>100)$, a Newton method is used which requires the computation of the Hessian of the objective function. An additional benefit of computing the Hessian is that the Hessian may be used to obtain a stochastic set of model coefficients in which a standard deviation of the computed model coefficients may be obtained. Details of computational algorithms and challenges are to be discussed in a forthcoming paper.

\section{Concluding Remarks}

This is a truly interdisciplinary project designed to resolve several very challenging research issues. Successful execution of this research requires collaboration and coordination across applied mathematics, computational science, physics, and biomedical engineering. Current progress is showing the mesh generation techniques and computational implementation of Pennes model to give good agreement with experimental MRTI data, Figure 4. 
The ability to combine Pennes model with a temperature dependent HSP expression model to be used as a predictive tool [6] for the optimal laser parameters for the laser treatment of cancer is also coming to realization.

Successful completion of this research will provide a powerful methodology for and insight into planning and optimizing the delivery of thermotherapy for cancer treatments. This study will also enable a better understanding of HSP kinetics and

Comparison Between Model Predicted and MRTI measured Temperature

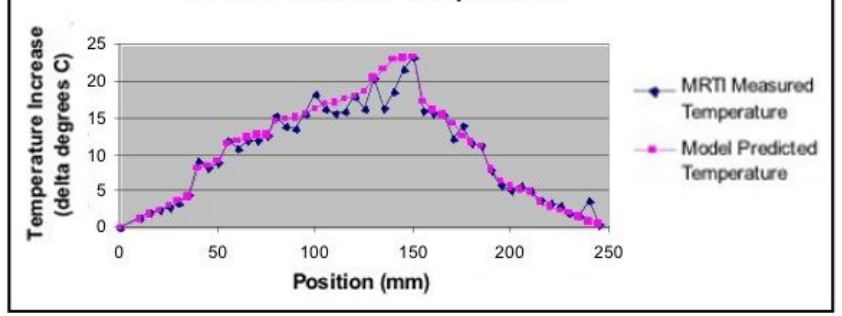

Fig. 4. Model Validation [5] damage profiles at the cellular and tissue levels in prostate cancer issued from metered thermal stress. The enormous breadth of discipline and the richness of the collaboration required in this effort will set a precedent.

Acknowledgments. The support of this project by the National Science Foundation under grant CNS-0540033 is gratefully acknowledged.

\section{References}

1. PROACT, Prostate Action Incorporated. http://www.prostateaction.org/statistics. http://www.cancer.org/docroot/STT/stt_0.asp.

2. J. Liu, L. Zhu, and L. Xu. Studies on the three-dimensional temperature transients in the canine prostate during transurethral microwave thermal therapy. J. Biomech. Engr, 122:372-378, 2000.

3. H. H. Pennes. Analysis of tissue and arterial blood temperatures in the resting forearm. J. Appl. Physiol., 1:93-122, 1948.

4. M. N. Rylander, Y. Feng, and K. R. Diller. Thermally induced HSP 27, 60, and 70 expression kinetics and cell viability in normal and cancerous prostate cells. Cell Stress Chaperones, In review, 2006.

5. M. N. Rylander, Y. Feng, J. Zhang, J. Bass, Stafford R. J., J. Hazle, and K. Diller. Optimizing hsp expression in prostate cancer laser therapy through predictive computational models. J. Biomed Optics, In press, 2006.

6. M.N. Rylander. Design of Hyperthermia Protocols for Inducing Cardiac Protection and Tumor Destruction by Controlling Heat Shock Protein Expression. PhD thesis, The University of Texas at Austin, 2005.

7. R. Salomir et al. Hyperthermia by MR-guided focused ultrasound: accurate temperature control based on fast MRI and a physical model of local energy deposition and heat conduction. Magn. Reson. Med., 43(3):342-347, 2000.

8. Y. Zhang and C. Bajaj. Adaptive and quality quadrilateral/hexahedral meshing from volumetric data. Computer Methods in Applied Mechanics and Engineering, 195:942-960, 2006. 